\title{
Channel Capacity of MIMO Architecture Using the Exponential Correlation Matrix
}

\author{
Sergey L. Loyka, Member, IEEE
}

\begin{abstract}
Multiple-input multiple output (MIMO) communication architecture has recently emerged as a new paradigm for wireless communications in rich multipath environment, which has spectral efficiencies far beyond those offered by conventional techniques. Channel capacity of the MIMO architecture in independent Rayleigh channels scales linearly as the number of antennas. However, the correlation of a real-world wireless channel may result in a substantial degradation of the MIMO architecture performance. In this letter, we investigate the MIMO channel capacity in correlated channels using the exponential correlation matrix model. We prove that, for this model, increase in correlation is equivalent to decrease in signal-to-noise ratio (SNR). For example, $r=0.7$ is the same as 3-dB decrease in SNR.
\end{abstract}

Index Terms-Channel capacity, correlation, MIMO.

\section{INTRODUCTION}

$\mathbf{M}$ ULTIPLE-INPUT multiple-output (MIMO) communication architecture has recently emerged as a new paradigm for wireless communications in rich multipath environment [1]-[4]. Using multi-element antenna arrays (MEA) at both transmitter and receiver, which effectively exploits the third (spatial) dimension in addition to time and frequency dimensions, this architecture achieves channel capacity far beyond that of traditional techniques. In independent Rayleigh channels the MIMO capacity scales linearly as the number of antennas under some conditions [1], [3], [4]. However, some impairments of the radio propagation channel may lead to a substantial degradation in MIMO performance. Some limitations on the MIMO capacity are imposed by the number of multipath components or scatterers [4]. Another limitation on the MIMO channel capacity, which is somewhat analogous to the multiple path limitation, is due to the correlation between individual sub-channels of the matrix channel [1], [5]-[7]. Increase in the correlation coefficient results in capacity decrease and, finally, when the correlation coefficient equals to unity, no advantage is provided by the MIMO architecture. The effect of fading correlation on the MIMO channel capacity has been investigated in details in [5] using an abstract one-ring scattering model. However, this approach does not allow to study the effect of correlation in an explicit way (i.e., capacity versus correlation).

Channel capacity of the two-antenna MIMO architecture has been investigated in [6] as an explicit function of the correlation coefficient. The general case of $n$-antenna architecture has been

Manuscript received April 16, 2001. The associate editor coordinating the review of this letter and approving it for publication was Prof. K. Kiasaleh..

The author is with the Department of Electrical Engineering, Ecole de Technologie Superieure, Montreal, QC H3C 1K3, Canada (e-mail: sergey.loyka@ieee.org).

Publisher Item Identifier S 1089-7798(01)08458-7. considered in [7] using the uniform correlation matrix model (when all the correlation coefficients are equal). This model may be used for the worst-case analysis or for some rough estimations using the average value of the correlation coefficient. However, the uniform model is somewhat artificial—one expects that the correlation of neighboring subchannels is higher than that of distant subchannels. In this way, we arrive to the exponential correlation model, which has been successfully used for many communication problems [8]-[10]. In this letter, we study the MIMO channel capacity using the exponential correlation matrix model by analytical techniques and derive a simple formula for the channel capacity indicating its validity range. We prove that, for this model, increase in correlation is equivalent to decrease in the signal-to-noise ratio under some realistic conditions. For example, $r=0.7$ is equivalent to the $3 \mathrm{~dB}$ decrease in SNR (as compared to the case of $r=0$ ). Finally, we compare this model with the uniform model and show that the exponential model predicts better MIMO performance.

\section{Channel CAPACity of MiMO ARChitecture}

For fixed linear $n \times n$ matrix channel with additive white Gaussian noise and when the transmitted signal vector is composed of statistically independent equal power components each with a Gaussian distribution and the receiver knows the channel, its capacity is [1]

$$
C=\log _{2} \operatorname{det}\left(\mathbf{I}+\frac{\rho}{n} \mathbf{H} \cdot \mathbf{H}^{+}\right) \text {bits } / \mathrm{s} / \mathrm{Hz}
$$

where $n$ is the number of transmit/receive antennas (for the sake of simplicity we consider here the case when the number of transmit and receive antennas are equal, but a general case can be considered in a similar way); $\rho$ is the average signal-to-noise ratio (SNR); $\mathbf{I}$ is $n \times n$ identity matrix; $\mathbf{H}$ is the normalized channel matrix, which is considered to be frequency independent over the signal bandwidth; and "+" means transpose conjugate. We adopt here the following normalization condition:

$$
\sum_{i, j=1}^{n}\left|h_{i j}\right|^{2}=n
$$

where $h_{i k}$ denotes the components of $\mathbf{H}$. Hence, when $\mathbf{H}=$ I (completely uncorrelated parallel sub-channels), $\rho / n$ is the signal-to-noise ratio per receive branch. Some other kinds of the normalization can also be used, but in this case $\rho / n$ will have a slightly different meaning.

To study the effect of correlation in an explicit way and to separate it from the effect of unequal received powers, we 
assume that all the received powers are equal. In this case, $\sigma_{i} \stackrel{\text { def }}{=} \sum_{j}\left|h_{i j}\right|^{2}=1$ and (1) reduces to [7]:

$$
C=\log _{2} \operatorname{det}\left(\mathbf{I}+\frac{\rho}{n} \mathbf{R}\right),
$$

where $\mathbf{R}$ is the normalized channel correlation matrix, $\left|r_{i j}\right| \leq 1$, whose components are:

$$
r_{i j}=\frac{1}{\sqrt{\sigma_{i} \sigma_{j}}} \sum_{k} h_{i k} h_{j k}^{*}=\sum_{k} h_{i k} h_{j k}^{*}
$$

where " $*$ " denotes complex conjugate. The last equality in (4) holds due to the assumption of equal received powers. It immediately follows from (4) that $r_{i j}=r_{j i}^{*}$.

It should be noted that (1)-(4) hold for a deterministic channel. When the channel is random (stochastic), the capacity is random, too. The mean (ergodic) capacity can be defined in this case [3]. Using the expectation over the channel matrix in (1), (2) and (4), we obtain the following upper bound on the mean (ergodic) capacity:

$$
\bar{C}=\langle C\rangle \leq C_{u p}=\log _{2} \operatorname{det}\left(\mathbf{I}+\frac{\rho}{n}\langle\mathbf{R}\rangle\right)
$$

where \langle\rangle is the expectation over the channel matrix. The inequality in (5) holds due to Jensen's inequality and concavity of logdet function [13]. Thus, our method will provide an upper bound on the mean capacity. For the sake simplicity, we assume further that the channel is deterministic, keeping in mind that the same results hold true for $C_{\text {up }}$ as well.

Denoting the matrix under the determinant in (3) by $\mathbf{Z}$, we obtain:

$$
\operatorname{det}(\mathbf{Z})=\left(1+\frac{\rho}{n}\right)^{n} \Delta_{n}
$$

where

$$
\Delta_{n}=\operatorname{det}\left[\begin{array}{ccccc}
1 & \beta r_{12} & \beta r_{13} & \cdots & \beta r_{1 n} \\
\beta r_{12}^{*} & 1 & \beta r_{23} & \cdots & \beta r_{2 n} \\
\beta r_{13}^{*} & \beta r_{23}^{*} & 1 & \cdots & \beta r_{3 n} \\
\cdots & \cdots & \cdots & \cdots & \cdots \\
\beta r_{1 n}^{*} & \beta r_{2 n}^{*} & \beta r_{3 n}^{*} & \cdots & 1
\end{array}\right]
$$

and

$$
\beta=\frac{\rho}{n}\left(1+\frac{\rho}{n}\right)^{-1} .
$$
form:

Thus, we may present the MIMO capacity in the following

$$
C=n \cdot \log _{2}\left(1+\frac{\rho}{n}\right)+\log _{2} \Delta_{n} .
$$

The first term is the MIMO capacity of $n$ parallel completely independent sub-channels [1] $\left(r_{i j}=0\right.$ in this case $)$ and the second term is the contribution of the sub-channel correlation. Note that the second term is negative (or zero) because $0 \leq \Delta_{n} \leq 1$. For high SNR $(\rho / n \gg 1), \beta \approx 1$ and $\Delta_{n}$ depends on the correlation matrix only. In fact, $\Delta_{n}$ is the Gram's determinant [11]. Thus, in order to estimate the MIMO capacity in a correlated channel, we need to evaluate $\Delta_{n}$.

\section{EXPONENTIAL CORRELATION MATRIX MODEL}

For this model, the components of $\mathbf{R}$ are given by

$$
r_{i j}=\left\{\begin{array}{cc}
r^{j-i}, & i \leq j \\
r_{j i}^{*}, & i>j
\end{array}, \quad|r| \leq 1\right.
$$

where $r$ is the (complex) correlation coefficient of neighboring receive branches (the correlation coefficient of signals in these receive branches). Obviously, (10) may be not an accurate model for some real-world scenarios but this is a simple single-parameter model which allows one to study the effect of correlation on the MIMO capacity in an explicit way and to get some insight. It remains to be investigated whether this model is applicable or not to some specific scenarios. Note, however, that this model is physically reasonable in the sense that the correlation decreases with increasing distance between receive antennas and it also corresponds to some realistic physical configurations (e.g., [9, p. 26]). Comparison with the recent measurement results [14] shows also that it provides reasonable conclusions when applied to a MIMO system.

Substituting (10) into (7) and after some equivalent transformations of the determinant (the second row multiplied by $r$ is subtracted from the first row, the third row multiplied by $r$ is subtracted from the second row etc.), we arrive to the following:

$$
\begin{aligned}
& \Delta_{n}= \\
& \operatorname{det}\left[\begin{array}{ccccc}
1-\beta|r|^{2} & (\beta-1) r & 0 & \cdots & 0 \\
\beta r^{*}\left(1-|r|^{2}\right) & 1-\beta|r|^{2} & (\beta-1) r & \cdots & 0 \\
\beta\left(r^{*}\right)^{2}\left(1-|r|^{2}\right) & \beta r^{*}\left(1-|r|^{2}\right) & 1-\beta|r|^{2} & \cdots & 0 \\
\cdots & \cdots & \cdots & \cdots & \cdots \\
\beta\left(r^{*}\right)^{n-1} & \beta\left(r^{*}\right)^{n-2} & \beta\left(r^{*}\right)^{n-3} & \cdots & 1
\end{array}\right] .
\end{aligned}
$$

A recurrent formula can be derived for $\Delta_{n}$ from (11) and be further used for its numerical evaluation (in this way, we reduce the computational time substantially as compared to the full matrix computation for large $n$ ). Unfortunately, it is not possible to derive a closed-form analytical expression from (11). But we can still obtain a simple closed-form expression in a practically-important case of high SNR $(\rho / n \gg 1)$, when $1-\beta \ll 1$. In this case, the main diagonal components only give the primary contribution to $\Delta_{n}$ :

$$
\Delta_{n} \approx\left(1-\beta|r|^{2}\right)^{n-1}
$$

and

$$
C \approx n \cdot \log _{2}\left(1+\frac{\rho}{n}\left(1-|r|^{2}\right)\right)+\log _{2}\left(\frac{1+\frac{\rho}{n}}{1+\frac{\rho}{n}\left(1-|r|^{2}\right)}\right) .
$$

In the case of $n \gg 1$ and $r<1$, we finally obtain:

$$
C \approx n \cdot \log _{2}\left(1+\frac{\rho}{n}\left(1-|r|^{2}\right)\right) .
$$

In the limiting case of $n \rightarrow \infty$, one obtains from (14):

$$
C_{\infty} \approx \frac{\rho}{\ln 2}\left(1-|r|^{2}\right) .
$$




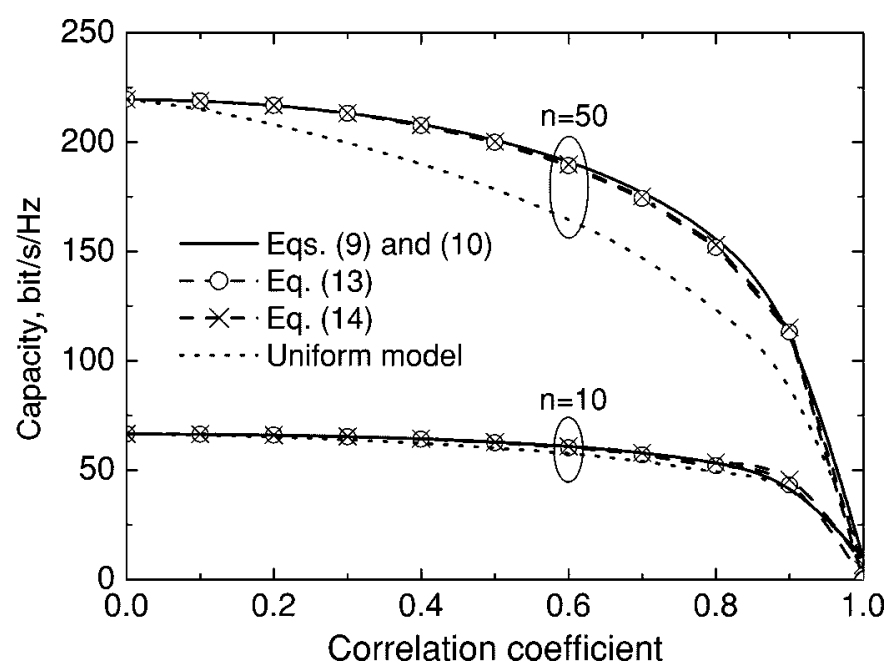

Fig. 1. MIMO channel capacity versus correlation coefficient, $\rho=30 \mathrm{~dB}$.

When $r=0$, the last two equations reduce to the well-known formulas (in this case, $\mathbf{H}=\mathbf{I}$ ) [1]:

$$
C=n \cdot \log _{2}\left(1+\frac{\rho}{n}\right) \text { and } C_{\infty}=\frac{\rho}{\ln 2} .
$$

Comparison of (14) and (15) with (16) clearly indicates that the effect of the channel correlation is equivalent to the loss in the SNR. Hence, for example, $r=0.7$ is equivalent to $3-\mathrm{dB}$ reduction in the SNR. Another possible interpretation of (14) and (15) is that the correlation of individual subchannels gives an increase in the noise level because for each particular sub-channel all the other subchannels are just the sources of interference (see, for example, the detection algorithm in [2]). Note also that the channel capacity does not depend on the correlation coefficient phase.

Fig. 1 shows the MIMO capacity of a deterministic channel versus the correlation coefficient evaluated by the full matrix computation [see (9) and (10)] and by approximate formulas (13) and (14) for $n=10$ and 50, and $\rho=30 \mathrm{~dB}$. The MIMO channel capacity evaluated using the uniform correlation matrix model [7] is also shown for comparison. As one may see from this figure, the accuracy of approximate formulas is quite good. It should be noted that the accuracy decreases as $n$ and $\rho / n$ decreases. The uniform model predicts lower capacity, as it should be (because it is the worst case model - the correlation between distant receive branches is the same as between neighboring ones). We also see that the MIMO capacity decreases significantly for $r>0.5$ to 0.8 , that agrees well with the known results on the spatial diversity techniques [12] and with recent measurements of the MIMO channel [14]. Detailed statistical analysis using Monte Carlo simulations of a correlated Rayleigh channel shows that its mean (ergodic) capacity is approximately $20 \%-40 \%$ smaller than that in Fig. 1 and it decreases with correlation in the same way as in the deterministic case.

\section{CONCLUSION}

The MIMO channel capacity depends substantially on the correlation between individual subchannels of the matrix channel, reaching its maximum value for zero correlation (completely independent parallel subchannels). In this letter, we have developed a method of MIMO capacity estimation using the correlation matrix approach. In particular, using the exponential correlation matrix model, we derived a simple formula for the MIMO channel capacity. The main conclusion is that the effect of channel correlation is the same as decrease in the signal-to-noise ratio under some realistic conditions. For example, $r=0.7$ is the same as 3-dB decrease in SNR. The exponential correlation matrix model predicts higher channel capacity than the uniform correlation matrix model, as it intuitively should be. While developed for a deterministic channel, our method provides an upper bound on the mean ergodic capacity of a stochastic channel as well.

\section{ACKNOWLEDGMENT}

The author wishes to thank Profs. Mosig and Rimoldi for discussions which inspired this work. The insightful comments of the reviewers are also appreciated.

\section{REFERENCES}

[1] G. J. Foschini and M. J. Gans, "On limits of wireless communications in a fading environment when using multiple antennas," Wireless Personal Commun., vol. 6, no. 3, pp. 311-335, Mar. 1998.

[2] G. D. Golden et al., "Detection algorithm and initial laboratory results using V-BLAST space-time communication architecture," Electron. Lett., vol. 35, no. 1, pp. 14-16, Jan. 7, 1999.

[3] I. E. Telatar, "Capacity of multi-antenna Gaussian channels," AT\&T Bell Labs., Internal Tech. Memo, June 1995.

[4] G. G. Rayleigh and J. M. Gioffi, "Spatio-temporal coding for wireless communications," IEEE Trans. Commun., vol. 46, pp. 357-366, Mar. 1998.

[5] D. S. Shiu et al., "Fading correlation and its effect on the capacity of multielement antenna systems," IEEE Trans. Commun., vol. 48, pp. 502-513, Mar. 2000.

[6] S. L. Loyka, "Channel capacity of two-antenna BLAST architecture," Electron. Lett., vol. 35, no. 17, pp. 1421-1422, Aug. 19, 1999.

[7] S. L. Loyka and J. R. Mosig, "Channel capacity of N-antenna BLAST architecture," Electron. Lett., vol. 36, no. 7, pp. 660-661, Mar. 2000.

[8] I. M. Kogan, Applied Information Theory. Moscow: Radio i Sviaz, 1981.

[9] G. L. Turin, "On optimal diversity reception," IRE Trans. Commun. Syst., vol. COM-10, pp. 22-31, Mar. 1962.

[10] P. Lombardo, G. Fedele, and M. M. Rao, "MRC performance for binary signals in Nakagami fading with general branch correlation," IEEE Trans. Commun., vol. 47, no. 1, pp. 44-52, Jan. 1999.

[11] Encyclopedia of Mathematics. Dordrecht, Germany: Kluwer Academic, 1989, vol. 4.

[12] W. C. Jakes, Jr., Microwave Mobile Communications. New York: Wiley, 1974.

[13] T. M. Cover and J. A. Thomas, Elements of Information Theory. New York: Wiley, 1991.

[14] C. C. Martin, J. H. Winters, and N. S. Sollenberger, "Multiple-input multiple-output (MIMO) radio channel measurements," in IEEE VTC'2000 Fall Conf., Boston, MA, Sept. 24-28, 2000. 